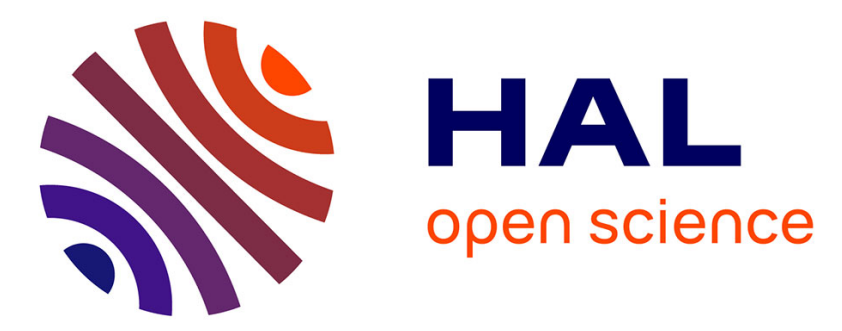

\title{
Probit latent variables estimation for a gaussian process classifier: Application to the detection of high-voltage spindles
}

\author{
Rémi Souriau, Vincent Vigneron, Jean Lerbet, Hsin-Chen Chen
}

\section{- To cite this version:}

Rémi Souriau, Vincent Vigneron, Jean Lerbet, Hsin-Chen Chen. Probit latent variables estimation for a gaussian process classifier: Application to the detection of high-voltage spindles. 14th International Conference on Latent Variable Analysis and Signal Separation (LVA/ICA 2018), Jul 2018, Guildford, United Kingdom. pp.514-523, 10.1007/978-3-319-93764-9_47 . hal-01825469

\section{HAL Id: hal-01825469 \\ https://hal.science/hal-01825469}

Submitted on 29 Jul 2019

HAL is a multi-disciplinary open access archive for the deposit and dissemination of scientific research documents, whether they are published or not. The documents may come from teaching and research institutions in France or abroad, or from public or private research centers.
L'archive ouverte pluridisciplinaire $\mathbf{H A L}$, est destinée au dépôt et à la diffusion de documents scientifiques de niveau recherche, publiés ou non, émanant des établissements d'enseignement et de recherche français ou étrangers, des laboratoires publics ou privés. 


\title{
Probit latent variables estimation for a Gaussian Process classifier. Application to the detection of High-Voltage Spindles
}

\author{
Rémi Souriau*, Vincent Vigneron, Jean Lerbet, and Hsin Chen \\ IBISC EA 4526, Université d'Evry, Université Paris Saclay, France \\ National Tsing Hua University, Electrical engineering department, Hsinchu, Taiwan \\ \{remi.souriau, vincent.vigneron, jean.lerbet\}@ibisc.univ-evry.fr \\ hchen@ee.nthu.edu.tw
}

\begin{abstract}
The Deep Brain Stimulation (DBS) is a surgical procedure efficient to relieve symptoms of some neurodegenerative disease like the Parkinson's disease (PD). However, apply permanently the deep brain stimulation due to the lack of possible control lead to several side effects. Recent studies shown the detection of High-Voltage Spindles (HVS) in local field potentials is an interesting way to predict the arrival of symptoms in PD people. The complexity of signals and the short time lag between the apparition of HVS and the arrival of symptoms make it necessary to have a fast and robust model to classify the presence of HVS $(Y=1)$ or not $(Y=-1)$ and to apply the DBS only when needed. In this paper, we focus on a Gaussian process model. It consists to estimate the latent variable $f$ of the probit model: $\operatorname{Pr}(Y=1 \mid$ input $)=\Phi(f($ input $))$ with $\Phi$ the distribution function of the standard normal distribution.
\end{abstract}

Keywords: Deep Learning, Gaussian Processes, Autoencoder, Classification, High-Voltage Spindle, Parkinson Diseases

\section{Introduction}

The Parkinson's disease (PD) is a progressive neurodegenerative disease. The depletion of the dopamine in the basal ganglia network leads to several symptoms like rigidity, posture instability, slow motion or pain for example. The expectation of the number of $\mathrm{PD}$ victims in Asian countries is 6.17 millions in 2030 [2]. The deep brain stimulation (DBS) is a surgical procedure used to relieve disabling neurological symptoms for diseases like PD [8]. A high-frequency stimulation signal (around $130 \mathrm{~Hz}$ ) is continuously applied to a deep-brain region called the subthalamic nucleus (STN) to relieve the symptoms. The main drawback of the DBS is the absence of any control on stimulation to minimize side effects. In addition, contemporary DBS implant requires another surgery to

\footnotetext{
* This work was partly supported by the National Tsing Hua University (Hsinchu, Taiwan) and Ministry of Science and Technology, R.O.C. (Taiwan).
} 
replace battery every 6 or 7 years.

Recent studies show we can predict the arrival of PD symptoms by the detection of high-voltages spindles (HVS) in recorded signals in local field potentials (LFPs) [1]. The HVS signals as e.g. in Fig. 1 are synchronous spike-and-wave patterns in LFPs oscillating in the $5-13 \mathrm{~Hz}$ frequency band. Suppressing HVS signals is found useful for delaying the progress of PD and deleting symptoms. Being able to detect HVS make possible the realization of a closed-loop system to control the DBS. However the diffusion of signals in the brain is nonlinear and there is only few milliseconds between the HVS wave and the apparition of PD symptoms. Hence a fast and robust model is needed for real time HVS detection and to apply the high frequency signal only when it is needed.

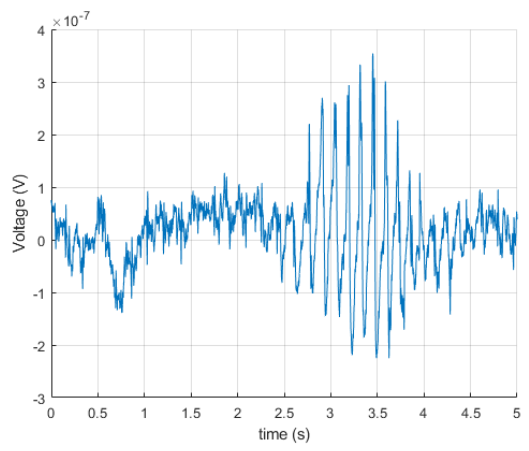

(a) Temporal representation.

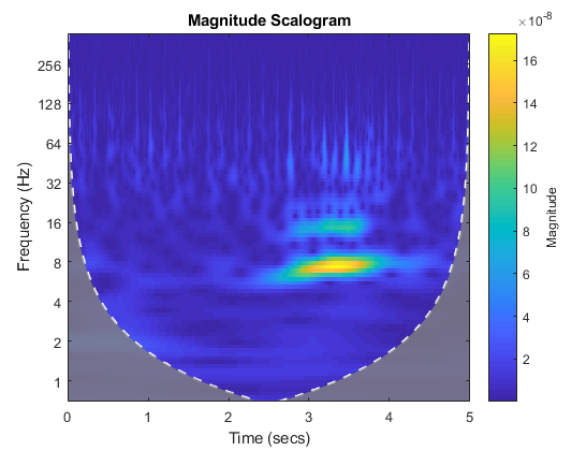

(b) Cosine wavelet transform using Morse wavelet.

Fig. 1: Signals recorded in LFPs in two different representations. HVS are located between 2.5 and 5 seconds. HVS are characterized by a fundamental frequency between 5 and $13 \mathrm{~Hz}$.

In this paper, the PD rat model is used. Data are collected from eight intracortical channels from different cortical regions. In this paper, we investigate performance of the Gaussian Process (GP) [3] for the detection of HVS. The GP model is a Bayesian network with continuous variables. Bayesian network model relations of causality between variables and in our study, data collected are the result of a diffusion of signals between neurons in the brain. Moreover, relations between variables model by a GP are nonlinear. Section 2 presents how data are collected and the preprocessing of data. Details of the model are developed in section 3. The two last sections give main results and discuss some future improvement and other possible approaches. 


\section{Data collection}

\subsection{Data Acquisition}

The PD rat model has been used to develop and evaluate the results. The description of the procedure to extract data is given in [7, sect. 2]. The LFPs were recorded from eight different brain regions listed in Tab.1. The frequency sampling of signals was $1 \mathrm{kHz}$ and the recording duration of one session was 60 seconds (60,000 samples). Several sessions have been recorded on PD rats.

\begin{tabular}{c|c} 
Notation & Region name \\
\hline M1D & Layer $5 b$ of the primary motor cortex \\
M1U & Layer $2 / 3$ of the primary motor cortex \\
M2D & Layer $5 b$ of the secondary motor cortex \\
M2U & Layer $2 / 3$ of the secondary motor cortex \\
SD & Layer $5 b$ of the primary somatosensory cortex \\
SU & Layer $2 / 3$ of the primary somatosensory cortex \\
STRI & Dorsal region of striatum \\
THAL & Ventrolateral thalamus
\end{tabular}

Table 1: List of brain region where LFPs signals were recorded.

\subsection{Data preparation}

GP classifier is a model which requires a supervised learning. The data preparation step consists to construct feature signals from collected data to train the model and feature signals for testing the model. One 60 s session has been used for the training step and the other session has been used for the testing step. The preprocessing step is the same for the two data sets. HVS wave's fundamental frequency is between 5 and $13 \mathrm{~Hz}$ and, according to observations in Fig. 1b, harmonics of HVSs are visible around $30 \mathrm{~Hz}$. High frequencies noises and continuous components of signals were deleted with a second order Butterworth filter between 1 and $200 \mathrm{~Hz}$ while preserving much of the HVS frequency content. Each channel was normalized independently from each other (zero mean and unit variance).

Then we define the prediction class vector $Y_{n} \forall n$ for the training set and the testing set. The presence of HVS is characterized by the apparition of spike-andwave patterns with a fundamental frequency between 5 and $13 \mathrm{~Hz}$. We estimate the Power Spectral Density (PSD) with a periodogram using the Hanning window with a $500 \mathrm{~ms}$ time window each $100 \mathrm{~ms}$. Then by computing the PSD mean between 5 and $13 \mathrm{~Hz}$ and performing an interpolation, we plot the PSD mean between $5-13 \mathrm{~Hz}$ as a function of time (see Fig. 2). We then defined our ground truth by thresholding our observations above at least one quarter of the signal magnitude. Result are given in Fig. 2. 

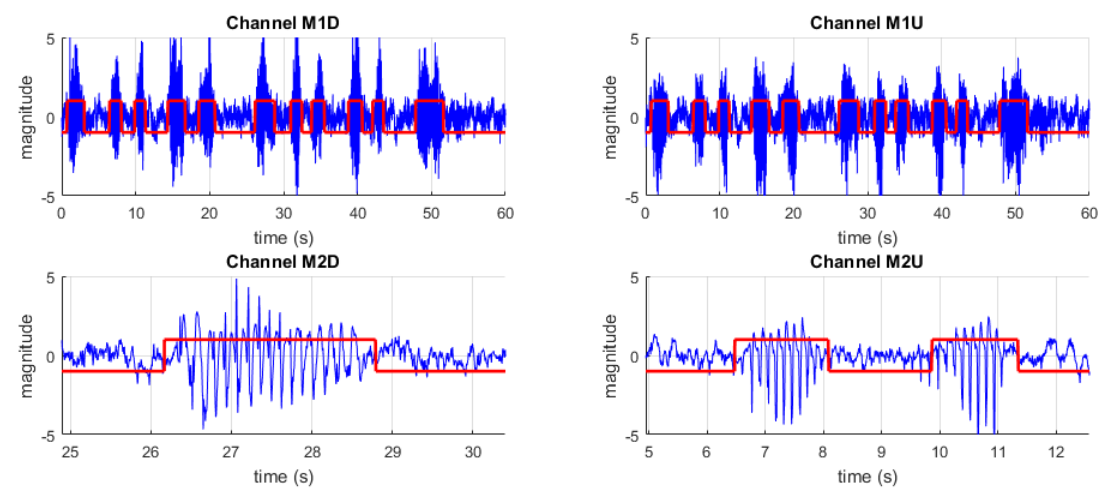

(a) PSD mean as a function of time for channels M1D, M2D, M1U and M2U.

The red line represent the ground truth: if $\frac{3}{4}$ of signal magnitude is above the threshold, then we consider we detect the presence of the HVS.
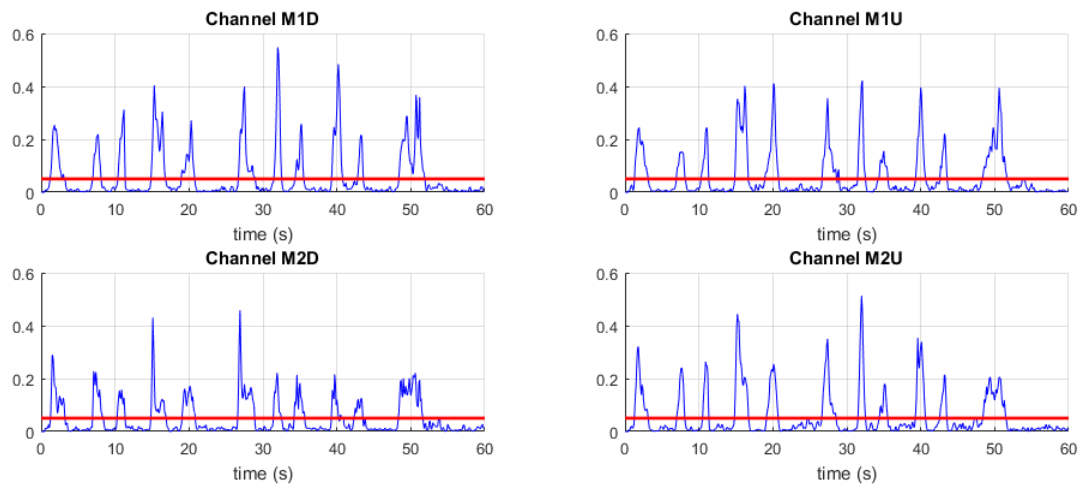

(b) Result of the classification on the four same channels. Channels MD2 and MU2 are zoomed to observe with more precision the switch of state HVS/no HVS and reverse.

Fig. 2: data preparation step. 


\section{Model}

\subsection{Gaussian process classifier}

A closed-loop DBS system delivers the stimulation only when needed.

Mathematically this consists to build a two-class classifier $\mathcal{C}$ capable to identify the presence or the absence of HVS. The available information for the classification are the values of the $R$ channels and $p$ previous signal values for each channel. Let $X_{n} \in \mathbb{R}^{R \times(p+1)}$ denotes the concatenated feature vector recorded between times $n$ and $n-p$ and $Y_{n} \in\{-1,1\}$ be the associated output of the supervised classifier. Suppose also the database is shared in a training set for which $Y_{n}$ is known and a test set for which $Y_{n}$ is unknown. The aim of the model is to estimate $Y_{n}$ from new observations $X_{n}$.

We note in the following $\mathcal{D}=\left\{X_{n}, Y_{n}\right\}_{n \in[1, N]}$ the training set with $X=$ $\left\{X_{n}\right\}_{n \in[1, N]}$ being independent randomly selected input observations and $Y=$ $\left\{Y_{n}\right\}_{n \in[1, N]}$ the associated output decision respectively. The GP classifier focus on modeling the posterior probabilities by defining the latent variables $f_{n}=f\left(X_{n}\right)$.

The model used here is the probit model: $\operatorname{Pr}(Y=1 \mid X)=\Phi(f(X))$ where $\Phi$ denotes the cumulative density function of the standard normal distribution. The likelihood of the probit model with independent observations and given $f=\{f(X(n))\}_{n \in[1, N]}$ is:

$$
p(Y \mid f)=\prod_{n=1}^{N} p\left(Y_{n} \mid f_{n}\right)=\prod_{n=1}^{N} \Phi\left(Y_{n} f_{n}\right) .
$$

In a GP, $f$ is a stochastic process which associates a zero mean normal random value for an input $X(n)$. For the training set $\mathcal{D}$ we have $p(f \mid X, \Theta) \sim \mathcal{N}\left(0, \mathbf{C}_{N}\right)$ where $\Theta$ is a set of hyper-parameters and $\mathbf{C}_{N}$ is a covariance matrix modelized with a squared exponential and a Gaussian noise [6]:

$$
\mathbf{C}_{N}\left(X_{i}, X_{j}\right)=\theta_{0}^{2} \exp \left(-\frac{1}{2} \sum_{n=1}^{\operatorname{dim}\left(X_{i}\right)} \frac{\left(X_{i}^{(n)}-X_{j}^{(n)}\right)^{2}}{\lambda_{n}^{2}}\right)+\theta_{1}^{2} \delta_{\left(X_{i}, X_{j}\right)} .
$$

$X_{i}^{(n)}$ is the $n$th component of $X_{i}$ and $\delta_{(\cdot)}$ is the Kronecker delta. The set of hyperparameters $\Theta$ is composed of $\left\{\theta_{1}, \theta_{2},\left\{\lambda_{n}\right\}_{n \in[1, N]}\right\}$. Baye's posterior probability rule of the latent variable $f$ with $\Theta$ known can be written:

$$
p(f \mid \mathcal{D}, \Theta)=\frac{p(Y \mid f) p(f \mid X, \Theta)}{p(\mathcal{D} \mid \Theta)}=\frac{\mathcal{N}\left(f \mid 0, \mathbf{C}_{N}\right)}{p(\mathcal{D} \mid \Theta)} \prod_{n=1}^{N} \Phi\left(Y_{n} f_{n}\right) .
$$

With the marginalization of Eq. (3) for a new observation $X_{N+1}$ we obtain:

$$
\operatorname{Pr}\left(f_{N+1} \mid \mathcal{D}, \Theta, X_{N+1}\right)=\int \operatorname{Pr}\left(f_{N+1} \mid f, X, \Theta, X_{N+1}\right) \operatorname{Pr}(f \mid \mathcal{D}, \Theta) \mathrm{d} f
$$


and the expectation of the Eq.4 gives:

$$
\operatorname{Pr}\left(Y_{N+1} \mid \mathcal{D}, \Theta, X_{N+1}\right)=\int \operatorname{Pr}\left(Y_{N+1} \mid f_{N+1}\right) \operatorname{Pr}\left(f_{N+1} \mid \mathcal{D}, \Theta, X_{N+1}\right) \mathrm{d} f_{N+1}
$$

We model the posterior probability $q(f \mid \mathcal{D}, \Theta) \sim \mathcal{N}(m, A)$ to compute $\operatorname{Pr}\left(Y_{N+1}=\right.$ $\left.1 \mid \mathcal{D}, \Theta, X_{N+1}\right)$. And then, for a new observation $N+1$, we can show that the posterior probability of $f_{N+1}$ is $q\left(f_{N+1} \mid \mathcal{D}, \Theta, X_{N+1}\right) \sim \mathcal{N}(\mu, \sigma)$ with:

$$
\begin{cases}\mu & =k^{T} \mathbf{C}_{N}^{-1} m \\ \sigma^{2} & =\kappa-k^{T}\left(\mathbf{C}_{N}^{-1}-\mathbf{C}_{N}^{-1} A \mathbf{C}_{N}^{-1}\right) k .\end{cases}
$$

where $k=\left(\mathbf{C}_{N}\left(X_{1}, X_{N+1}\right), \ldots, \mathbf{C}_{N}\left(X_{N}, X_{N+1}\right)\right)^{T}$ is the covariance function vector between each observation of the training set and the new observation $X_{N+1}$ and $\kappa=\mathbf{C}_{N}\left(X_{N+1}, X_{N+1}\right)=\theta_{0}^{2}+\theta_{1}^{2}$ is the variance of $X_{N+1}$.

With the approximation of $\operatorname{Pr}(f \mid \mathcal{D}, \Theta)$, Eq. (5) becomes:

$$
\operatorname{Pr}\left(Y_{N+1}=1 \mid D, \Theta, X_{N+1}\right)=\Phi\left(\frac{\mu}{\sqrt{1+\sigma^{2}}}\right)
$$

Training a GP consist to find $\Theta, m$ and $A$. We can learn $\Theta$ by computing the $\log$-likelihood of the $\log$ posterior probability $\log q(Y \mid X, \Theta)$ Eq.8 (see [6, Chapter $5])$ and his gradient in function of $\Theta$.

$$
\log q(Y \mid X, \Theta)=-\frac{1}{2} f^{T} \mathbf{C}_{N}^{-1} f+\log p(Y \mid f)-\frac{1}{2} \log \operatorname{det}\left(I+W^{\frac{1}{2}} \mathbf{C}_{N} W^{\frac{1}{2}}\right)
$$

With $W=-\Delta_{f} \log p(Y \mid f)$ (Hessian) and $f$ such the unormalized log likelihood $\log p(f \mid \mathcal{D}, \Theta)$ is maximized:

$$
\begin{aligned}
\log p(f \mid \mathcal{D}, \Theta) & =\log p(Y \mid f)+\log p(f \mid X) \\
& =\log p(Y \mid f)-\frac{1}{2} f^{T} \mathbf{C}_{N}^{-1} f-\frac{1}{2} \log \operatorname{det}\left(\mathbf{C}_{N}\right)-\frac{N}{2} \log 2 \pi
\end{aligned}
$$

For a given $\Theta$, we can find $m=\arg \max _{f} \log p(f \mid \mathcal{D}, \Theta)$ by using the Newton's method. Eq.10 and Eq.11 give the gradient and the hessian of $\log p(f \mid \mathcal{D}, \Theta)$, respectively.

$$
\begin{gathered}
\nabla_{f} \log p(f \mid \mathcal{D}, \Theta)=\nabla_{f} \log p(Y \mid f)-\mathbf{C}_{N}^{-1} f . \\
\Delta_{f} \log p(f \mid \mathcal{D}, \Theta)=\Delta_{f} \log p(Y \mid f)-\mathbf{C}_{N}^{-1} .
\end{gathered}
$$

The maximization of $\log p(f \mid \mathcal{D}, \Theta)$ makes use of the first and second order partial derivation of $\log p(Y \mid F)$ in function of $f_{i}$.

$$
\begin{gathered}
\frac{\partial}{\partial f_{i}} \log p(Y \mid f)=\frac{Y_{i} \phi\left(f_{i}\right)}{\Phi\left(Y_{i} f_{i}\right)} . \\
\frac{\partial^{2}}{\partial f_{i}^{2}} \log p(Y \mid f)=-\frac{\phi\left(f_{i}\right)^{2}}{\Phi\left(Y_{i} f_{i}\right)^{2}}-\frac{Y_{i} f_{i} \phi\left(f_{i}\right)}{\Phi\left(Y_{i} f_{i}\right)} .
\end{gathered}
$$


Where $\phi($.$) is the density function of the standard normal distribution. Learning$ $m$ allow to compute Eq.8 and their gradient in function of $\Theta$. We implement a gradient descent search of the optimum $\Theta^{*}$ that leads to the following iterative algorithm:

$$
\left.\Theta^{(k+1)}=\Theta^{(k)}-\alpha_{k} \nabla_{\Theta} \log q(Y \mid X, \Theta)\right) .
$$

But Eq. (14) requires to inverse the $N \times N$ matrix $\mathbf{C}_{N}$ at each iteration which can be time consuming for a large number of observations. Once $m$ and $\Theta$ found, we can compute $A=\left(\mathbf{C}_{N}^{-1}+W\right)^{-1}$.

Finally, the GP classifier is learned by identifying the covariance matrix between observations $\mathbf{C}_{N}$ as a function of the hyper-parameters $\Theta$, the mean vector $m$ is learning for each iteration of $\Theta$ then and the covariance matrix $A$ is deduced. Once the learning is done, the prediction step consists to compute the covariance vector $k$ between the new observation $X_{N+1}$ and the training set $X$ and then estimates the probability $\operatorname{Pr}\left(Y_{N+1}=1 \mid \mathcal{D}, \Theta, X_{N+1}\right)$. If $\operatorname{Pr}\left(Y_{N+1}=1 \mid \mathcal{D}, \Theta, X_{N+1}\right)>$ 0.5 then $Y_{N+1}=1$ and $Y_{N+1}=-1$ else.

\subsection{Input autoencoding}

Learning the model consist in two step: learning the hyper-parameters $\Theta$ and learning the parameters of $q\left(f_{N+1} \mid \mathcal{D}, \Theta, X_{N+1}\right)$. HVS have a fundamental frequency between 5 and $13 \mathrm{~Hz}$. with a the maximal period of $200 \mathrm{~ms}$. Choosing $p=199$ to have at least one period of the signal leads to a model with high dimensions: the input size of $X_{n}$ is then $(p+1) \times R=1600$ and $\Theta$ has 1602 parameters. To reduce the the dimensionality of the input vector $X_{n}$ (which makes it difficult to use for real time applications) we use an autoencoder (see Fig. 3). This autoencoder consists in a 3 layers neural network that compresses input data onto the hidden layer. We present to the input and the output layers the same input vector $X_{n}$. The activation function of the hidden layer is sigmoïdal function $s(\cdot)$ that permits nonlinear combination of the inputs:

$$
s\left(x_{j}\right)=\frac{1}{1+\exp \left(-b_{j}-\sum_{i} w_{i j} x_{i}\right)}
$$

where $\left(x_{1}, \ldots, x_{p}\right)^{T}$ is the input vector. Learning this autoencoder consists to find biases $b_{j}$ and weights $w_{i j}$ of the input neurons. The output layer has to be the closest possible to the input layer. The training algorithm is the scaled conjugate gradient [4] using the mean square error with $L_{2}$ sparsity regularized loss function [5].

Fig. 3 gives an example of result for a number of observations $N=500$ and the size of the autoencoder $H=10$. The sensitivity and the specificity (see section 4) are, respectively, $82.92 \%$ and $99.31 \%$.

\section{Experimental results}

Detection of HVS has been applied on different rats with various set of parameters for the learning stage such as the number of observations $N$ or the size of 
the hidden layer of the autoencoder $H$.

The small the parameters, the lower the number of parameters: learning the model and use it become very fast by reducing the dimensionality. Choosing $N$ small means taking the risk to not have enough observations or have observations not sufficiently representative.

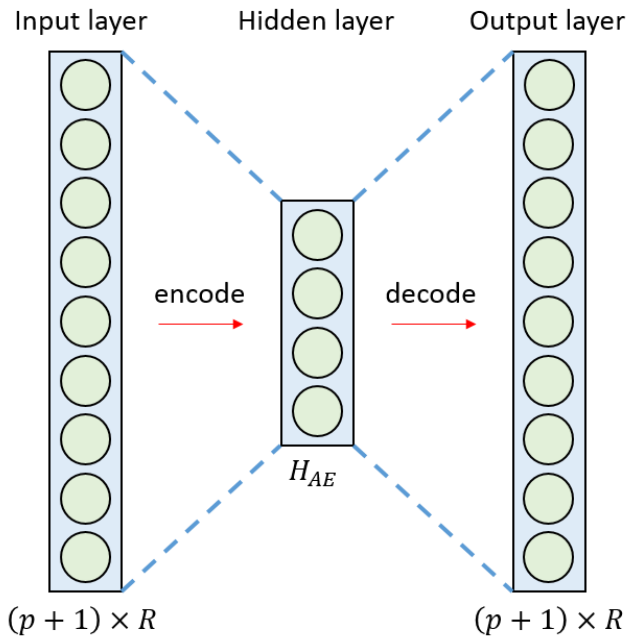

(a) Autoencoder architecture.
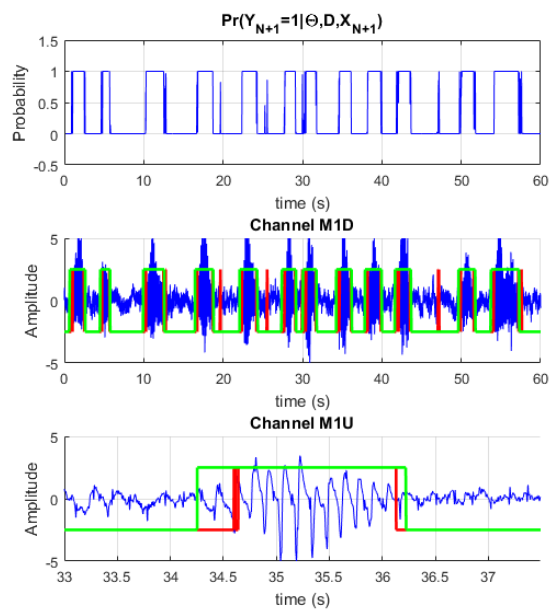

(b) Example of result after training the model autoencoder + Gaussian Process.

Fig. 3: In (3a) autoencoder neural network: $(p+1) \times R$ corresponds to the dimension of the input and the output layers and $H_{A E}$ is the size of the hidden layer (parameters). In (3b), the plot to the top $\operatorname{Pr}\left(Y_{N+1}=1 \mid \mathcal{D}, \Theta, X_{N+1}\right)$ in function of the time. The two other figures are two among five channels of the testing set. Green line is the ground-truth defined in the preprocessing step. The red line is the decision made by the GP classifier. The second channel is zoomed on a HVS.

For each rat, one signal session record has been used for the learning step and an other session has been used for the testing step. The criteria of performance for models are the sensitivity $S e=T P /(T P+F N)$ and the specificity $S p=$ $T N /(T N+F P)$, with $T P$ is the number of true positive, $T N$ is the number of true negative, $F N$ is the number of false negative and $F P$ is the number of false positive. The sensitivity gives the true positive rate: the number of correct detection under the number of correct detection and miss. The specificity give the true negative rate: the number of correct non detection under the number of correct non detection and false detection. We reproduce 5 times for each set of parameters the learning and the testing stages and compute the mean and the variance of $S e$ and $S c$ to verify the performance and the robustness, regarding 
random sampling.

Results are summarized in Tab.2. Data collection for each rat is different: the rat 2 provides data from channels M1U, M1D, SU and SD; rat 3 provides data from channels M1U, M1D, STRI and SD; rat 1 provides data from all channels ${ }^{1}$. Results are discuss in next section.

\begin{tabular}{|c|c|c|c|c|c|c|c|c|c|c|c|c|c|}
\hline \multirow{3}{*}{$N$} & \multirow{3}{*}{$H$} & \multicolumn{4}{|c|}{ Rat number 1} & \multicolumn{4}{|c|}{ Rat number 2} & \multicolumn{4}{|c|}{ Rat number 3} \\
\hline & & Sefi & & & & Sonci & & & & on & & & fici \\
\hline & & and & & ean & & $m$ & & $\mathrm{~m}$ & & & & me & \\
\hline 50 & 10 & .58 & & .88 & & & & .47 & & .23 & & .83 & \\
\hline 50 & & & & & & & & & & & & & \\
\hline 50 & 50 & .62 & & .97 & & & & .52 & & .12 & & .91 & \\
\hline 50 & 100 & & & & & .4 & & .78 & & .10 & & .93 & 004 \\
\hline & & & & & & & & & & & & & \\
\hline & & & & & & & & .7 & & & & .8 & 1. \\
\hline & & .81 & & .91 & & .71 & & 73 & & .13 & & .90 & \\
\hline & 100 & & & .9 & & & & .82 & & .01 & & .99 & 00 \\
\hline & 10 & & & & & .6 & & .65 & & .14 & & .94 & V \\
\hline & & & & & & .7 & & .67 & & .25 & & .8 & 0 \\
\hline & 50 & .83 & & .89 & & 7 & & .68 & & .21 & & .85 & .01 \\
\hline 500 & 100 & .74 & .0226 & .94 & .0054 & .76 & .0029 & .70 & .0005 & .85 & .0032 & .85 & .002 \\
\hline
\end{tabular}

Table 2: Result of the experience. $N$ is the number of observations used from the training set. $H$ is the size of the hidden layer of the encoder. Variance equal to .0000 in the table mean the value is less than $10^{-4}$. Bold numbers highlight most relevant results.

\section{Conclusion}

Tab.2 highlights some tendencies in the parameters. First, the number of observations is critical for fine sensitivity and specificity. Taking too little oservation can alter the overall knowledge of the system : in this case the variance is often more important for $N=50$ than for bigger $N$. But too much data leads to big with covariance matrices too long to calculate. Increasing $H$ (hidden layer number of neurons) increases the sensitivity but decreases the specificity: the model tends to detect HVS all the time. On the opposite for small $H$ we compress a lot of data by taking the risk to loose information. A large hidden layer better preserves the information but $(i)$ the problem becomes hard to optimize (too much parameters) (ii) learning the model become time-consuming.

Results for rat 2 and 3 are not as fine as the first rat. By looking closely step by step signals of the two rats it appear that the intensity of the noise is much more important than in signals of the first rat. Means over channels of signal-toratio of the three rats are respectively, 35 decibels, 14 decibels and 10 decibels.

\footnotetext{
${ }^{1}$ see Tab.1 as a reminder.
} 
Moreover, in rat 2 and 3, appearance of signals differs according to the various channels: some HVS do not appear in all channel which make the preprocessing step not relevant for those two rats. This is why results of rat 2 and 3 are not reliable to conclude with a high confidence level about the robustness of the model.

In a future work, we will develop an approach based on unsupervised learning model because by defining ourselves the groundtruth we may have missed some complex features in the signal which could have helped for predicting HVS. Restricted Boltzmann Machines is a promising stochastic model which, by exploring latent variables could find such hidden features.

\section{References}

1. Cyril Dejean, Christian E Gross, Bernard Bioulac, and Thomas Boraud. Dynamic changes in the cortex-basal ganglia network after dopamine depletion in the rat. Journal of neurophysiology, 100(1):385-396, 2008.

2. ERl Dorsey, R Constantinescu, JP Thompson, KM Biglan, RG Holloway, K Kieburtz, FJ Marshall, BM Ravina, G Schifitto, A Siderowf, et al. Projected number of people with parkinson disease in the most populous nations, 2005 through 2030. Neurology, 68(5):384-386, 2007.

3. Malte Kuss and Carl Edward Rasmussen. Assessing approximate inference for binary gaussian process classification. Journal of machine learning research, 6(Oct):1679-1704, 2005.

4. Martin Fodslette Møller. A scaled conjugate gradient algorithm for fast supervised learning. Neural networks, 6(4):525-533, 1993.

5. Bruno A Olshausen and David J Field. Sparse coding with an overcomplete basis set: A strategy employed by v1? Vision research, 37(23):3311-3325, 1997.

6. Carl Edward Rasmussen. Gaussian processes in machine learning. In Advanced lectures on machine learning, pages 63-71. Springer, 2004.

7. Vincent Vigneron, Tahir Qasim Syed, and Hsin Chen. Automatic detection of highvoltage spindles for parkinson's disease. In BIOSIGNALS, pages 372-378, 2015.

8. Jerrold L Vitek. Mechanisms of deep brain stimulation: excitation or inhibition. Movement disorders, 17(S3), 2002. 